\title{
Bridging the Degeneration in Public Cultural Services Due to Policy Implementation Bias in the Context of Information Technology
}

\author{
Tingting $\mathrm{Li}^{1}{ }^{1,}$ Jiani $\mathrm{Jin}^{1,}$ Xiaoning $\mathrm{ZHU}^{1, *}$ \\ ${ }^{1}$ School of Public Administration, University of Electronic Science and Technology, Chengdu, 611731, China \\ "Corresponding author. Email: zhuxn1956@163.com
}

\begin{abstract}
Public cultural service degeneration is the degeneration between the supply of public cultural services and people's demand for public cultural services. The formation of the degeneration in public cultural services is influenced by many factors, but this paper focuses its analysis on policy implementation bias and investigates the causes of the degeneration in public cultural services based on the four dimensions of Edward's policy implementation model: communication, resources, implementer preferences, and bureaucratic structure. The study finds that poor policy communication, shortage of policy resources, selfish preferences of the implementing agents, and internal weaknesses of the bureaucracy all contribute to the creation of public cultural degenerations. Finally, it is suggested that big data can be used to precisely match the cultural needs of the public and better bridge the degeneration in public cultural services, so as to improve the supply of public cultural services and achieve a balance between the supply of government public cultural services and the needs of citizens.
\end{abstract}

Keywords: Edward Policy Implementation Model, Digital Governance, Matching Supply and Demand for Public Cultural Services.

\section{INTRODUCTION}

Since the release of the National Cultural Development Plan for the Eleventh Five-Year Plan period in 2006, the pace of construction of China's public cultural service system has been accelerating, and the Law of the People's Republic of China on the Protection of Public Cultural Services was promulgated in December 2016, which stipulates that special groups such as minors, the elderly, people with disabilities and the floating population are included in the public cultural service system. In March 2017, the 13th FiveYear Plan to Promote the Equalization of Basic Public Services was released, and the implementation of menu services to match supply and demand is one of the six key tasks in the deployment of public cultural services. At the 19th Party Congress, it was mentioned that "to meet the people's new expectations for a better life, we must provide rich spiritual food. Continuously improve the public cultural service system, in-depth implementation of cultural projects for the benefit of the people, and enrich cultural activities for the masses." [1] From the content of the above policies, it can be seen that the development of public cultural services not only emphasizes public welfare, equality and convenience, but also begins to pay more attention to the people's personalized and precise public cultural service needs. However, in reality, the supply of public cultural services is single, sloppy and unbalanced, and there is a degeneration between the public demand for public cultural services and the public demand for public cultural services, which means that public cultural services are degraded. Therefore, it is of great theoretical and practical significance to investigate the causes of the degeneration in public cultural services, to solve the problems of resource mismatch and disparity between supply and demand, to improve the efficiency and level of public cultural services, to truly realize the cultural benefits for the people, and to promote the modernization of national governance in the field of culture. However, the research on public cultural service degeneration is still at the preliminary stage, and there is still some discussion on "what is public cultural service degeneration", "what causes public cultural service degeneration" and "how to bridge the degeneration". This study has not yet conducted any systematic and in- 
depth research on "what is the degeneration in public cultural services," "what causes the degeneration in public cultural services," and "how to bridge the degeneration in public cultural services. Thus, this study attempts to use Edward's policy implementation model to investigate the causes of the degeneration in public cultural services in four dimensions: communication and exchange, resources, implementer preferences, and bureaucratic structure; and finally, to explore countermeasures and suggestions to better bridge the degeneration in public cultural services from the perspective of big data-assisted governance to improve the efficiency and level of public cultural service provision.

\section{AWARENESS OF " CULTURAL DEGRADATION "}

In his book Social Change: On Culture and Nature, American sociologist W.F. Oppenberg first introduced the concept of cultural degeneration, which he defined as a phenomenon in which "changes in non-material culture in social change are always later than changes in material culture". [2] In other words, in the process of social change, what develops faster is science and technology and material culture, while the development of concepts and institutional systems lags behind, which gives rise to cultural degeneration. On this basis, domestic scholars have carried out relevant studies based on cultural distance, and proposed "institutional degeneration", [3] "governmental function degeneration", [4] "public cultural service degeneration", [5] and so on, and have been developing new research fields. The theoretical viewpoints have been reviewed, and the term " degeneration" refers to the degeneration between what is made possible and what should be made possible due to delayed or lagging actions. Therefore, this paper defines the degeneration of public cultural services as the degeneration between the government's supply of public cultural services and the public's demand for public cultural services. [6]

\section{EVALUATION OF THE EFFECT OF POLICY IMPLEMENTATION}

All of the studies in this paper are based on the assumption that public cultural policy is an ideal policy and the realization of public cultural policy goals is equivalent to satisfying the public's demand for public cultural services. Deviations in the implementation of public cultural service policy will result in the failure to achieve the policy goal and the failure of public cultural service supply to meet the public's demand for public cultural services, resulting in the creation of a public cultural service degradation.

Although the quality and level of public cultural services in China have been steadily improving, there are still various problems in the supply of public cultural services. In addition to the factors of policy formulation and policy environment, the deviation in the implementation of public cultural policies is an important reason for the abortion distance of public cultural services. According to Chen Qingyun, the only way to transform policy ideals into policy realities and policy goals into policy benefits is policy implementation, which is one of the most important links in achieving policy goals. [7] Therefore, the article has theoretical and practical significance in applying Edward's policy implementation model, analyzing the influencing factors of public cultural service policy implementation, reviewing the effect of public cultural policy implementation, and exploring the countermeasure suggestions of big data to assist in solving the abortion distance of public cultural service.

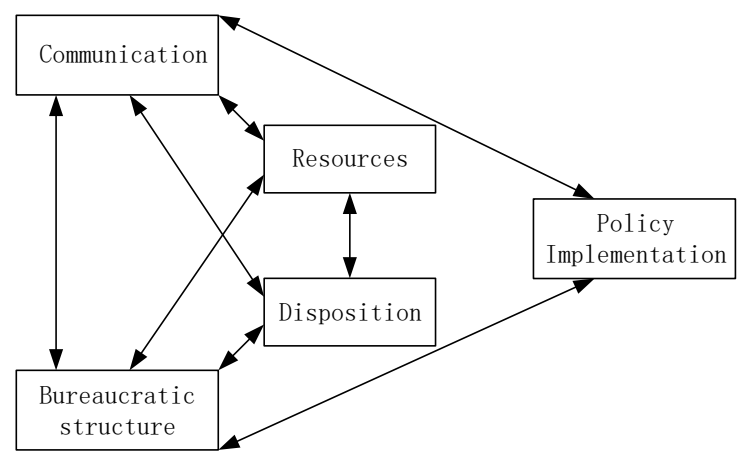

Figure 1 Edward's Policy Implementation Model

In his book Implementing Public Policy, G. Edward presents the Edward Model of Policy Implementation, as shown "Fig. 1", which states that the interaction of four main factors: communication and exchange, resources, policy implementer preferences, and bureaucratic structure directly or indirectly affects policy implementation. Communication and communication include communication about policy implementation between upper and lower levels of government, and between governments and people; resources include human, material, and financial resources needed to implement policies; implementer preferences include the implementer's interest preferences for the policy, the level of support, and the overall quality of the individual implementer; and bureaucratic organizational structure is mainly manifested in internal organizational structure and division of responsibilities.

\subsection{Poor policy communication and mismatch between supply and demand of cultural services}

The implementation of public cultural policies may be distorted and perfunctory by policy implementers due to poor communication between upper and lower levels of government. In order to respond to the policies of the higher levels, local governments arrange activities such 
as "cultural and artistic activities in the countryside", but the policy implementers and performers carry out the activities with the perfunctory mentality of "finishing the performance and then being done with it", and the villagers are not satisfied with most of the cultural performances. The villagers were not satisfied with most of the cultural performances, and the cultural implementation failed to achieve the expected results.

Without a deeper understanding of the people's needs for cultural services, the implementation of public cultural policies often results in "wishful thinking" and fails to meet the diverse and individual needs of the people.

\subsection{Shortage of policy resources and uneven supply of cultural services}

The implementation of public cultural policies requires the support and guarantee of policy resources such as human, material and financial resources, and the shortage of policy resources in the actual implementation process has become a negative constraint affecting the effectiveness of public policy implementation.

The inconsistency of socio-economic development between regions leads to the disparity of human, financial and other resources invested in the implementation of public cultural policies, resulting in the "unbalanced and inadequate" supply of public cultural services among regions, urban and rural areas, and audience groups, forming the "Matthew effect" in the supply of public cultural services. The "Matthew effect" of public cultural service provision [8]. On the other hand, the development of public cultural services in the central and western regions and rural areas, which are "culture deficient", has been slow, and it is difficult to implement public cultural services even with the keynote of "maintaining the basics", and the balance of public cultural services has not been effectively implemented.

\subsection{Preference of implementation subjects and poor cultural service supply}

Public cultural policy itself is the regulation of social interests, and policy implementation is the process of interest game among subjects and the pursuit of their own interest maximization. [9] If the adjustment of policy implementation affects the scope of interests of each subject, each implementing subject may put its own local or personal interests above the overall interests driven by interests, and the cultural interests of the public cannot be satisfied.

The attitude of implementers toward public cultural policies and the comprehensive quality of the implementers themselves will affect the implementation effect of public cultural policies. Insufficient awareness or misconceptions of the implementers will lead to negative and resistant attitudes toward public cultural policies, which will lead to symbolic implementation and selective implementation in the process of policy implementation; and the low cultural and theoretical level of policy implementers will lead to wrong implementation of policies, which will eventually lead to deviations in the implementation of public cultural policies and affect the effectiveness of public cultural service policies. The implementation effect of public cultural service policy is affected.

\subsection{Deficiencies of bureaucracy and low efficiency of cultural service provision}

The deficiencies of policy implementation agencies are mainly reflected in two aspects: one is the unreasonable organizational structure. Public cultural service policy departments are intertwined and contradictory in terms of departmental barriers. Vertically, there are large differences in the institutional settings of cultural and tourism departments at different levels, and the correspondence between upper and lower institutions is unclear. [10] Horizontally, the implementation of public cultural policies often also involves multiple policy departments such as education and finance, and these departments often have multiple heads and lack of coordination in the actual policy implementation process, making the implementation of public cultural policies less effective.

On the other hand, the lack of supervision, assessment, and accountability of policy implementation agencies may result in the implementation of public cultural policies in a fragmented manner in pursuit of maximizing their own interests, resulting in conflicts of interest that affect the implementation of public cultural policies and deviate from the target direction set by the policies, making it difficult to meet the target requirements of public cultural services.

\section{SUGGESTIONS}

\subsection{Broaden information and communication channels}

First, policy implementers should strengthen communication with higher governments to clarify policy goals and intentions, so that they can more accurately decompose and refine policies when they are discretionary, and thus better implement public cultural policies.

Secondly, policy implementers should strengthen communication with policy audiences to understand people's real needs for public cultural services, make accurate and targeted supply, realize effective matching between supply and demand, better meet people's needs 
for public cultural services, and bridge the abortion gap of public cultural services.

Finally, in order to achieve better communication and exchange, it is necessary to rely on big data to innovate the way of communication and exchange. By building a government intranet to achieve information sharing within the government; the government can use the government service platform to achieve communication between the government and citizens, the public's demand for public cultural services can be reflected on the platform in real time, and policy makers and implementers can make timely adjustments according to the public's demand; at the same time, according to the public's demand information, the public's demand for public cultural services can be portrayed by means of big data algorithms, etc., so as to accurately and effectively supply public cultural services. Thus, public cultural services can be supplied precisely and effectively, and the lag in the implementation of public cultural policies can be reduced to a certain extent, and the implementation effect of public cultural policies can be enhanced.

\subsection{Coordinating policy resources}

Adequate policy resources can strongly promote the effective implementation of policies, and the coordination and coordination of human, material and financial resources are essential in the provision of public cultural services.

In terms of human resources, we should analyze the shortage of grass-roots personnel in public cultural services based on big data, and plan to increase the number of grass-roots personnel by establishing a longterm mechanism for the construction of grass-roots personnel; in response to the shortage of personnel in "culture deficient" regions, we should actively attract social forces to join in the supply of public cultural services, so as to effectively alleviate the uneven supply of public cultural services due to the lack of personnel.

In terms of material and financial resources, the data analysis will help the central government and local governments at all levels to precisely increase the supply of financial budgets and special funds for cultural services to the less developed areas, so as to better achieve the policy goal of "full coverage" of public cultural services.

\subsection{Improve the quality of policy implementation personnel}

By building an implementation supervision information system to achieve real-time monitoring of the policy implementation process, the implementation subjects will automatically upload their implementation behaviors and effects into the information system in the implementation process, which will enable real-time tracking and evaluation of the implementation status of the implementation subjects, achieving the purpose of strengthening supervision and better promoting the effective implementation of public cultural policies [12].

Improving the comprehensive quality of implementers should improve the ideological and moral quality of policy implementers, put the interests of the people in the highest position, and prevent the ideas and behaviors of using power for personal gain and violating the interests of the people; meanwhile, it should strengthen the professional training of implementers in public cultural services, broaden their knowledge, enhance their understanding and analysis of policies, and better implement public cultural policies.

\subsection{Improve internal bureaucratic institutions}

Establish a multi-departmental linkage mechanism and data and information sharing platform. Vertically, it compresses layers and improves the efficiency of policy implementation; horizontally, it reduces information barriers and coordinates and integrates multiple resources among departments to better realize the implementation of public cultural policies.

Relying on the implementation monitoring information system, a proven accountability system has been established for each policy implementation body and policy internal agencies. By clarifying the responsibilities and obligations between internal agencies and policy implementation bodies, and holding departments and bodies responsible for policy implementation errors, we can better constrain policy implementation departments and bodies to implement public cultural policies with high quality, meet people's demand for public cultural services, and bridge the gap between public cultural services.

\section{CONCLUSION}

This article uses Edward's policy implementation model to analyze the causes of the gap in public cultural services in four dimensions: communication and exchange, resources, implementer preferences, and bureaucracy; it concludes that poor policy communication, shortage of policy resources, selfinterest of implementers, and internal defects of bureaucracy all contribute to the gap in public cultural services. Finally, the article proposes countermeasures for bridging the gap in public cultural services in terms of widening communication channels, coordinating policy resources, improving the quality of personnel, and improving bureaucratic institutions.

In the future, research on the causes of public cultural service disparity can be conducted from the perspective of policy process, and the causes of public 
cultural service disparity can be analyzed from three dimensions: policy formulation, policy implementation, and policy evaluation; at the same time, research can also focus on bridging public cultural service disparity and continue to study public cultural service disparity in depth.

\section{REFERENCES}

[1] "Compilation of documents of the 19th Party Congress" [G]. Beijing:Party Building Readers Publishing House, 2017:30;8.

[2] Liao Gelong,Sun Liancheng,Chen Youjin,et al. Encyclopedia of Marxism (next volume) [M]. Beijing: People's Daily Press, 1993 : 1635,1636.

[3] Xin Qiushui. "Institutional degradation distance and institutional improvement--a study report on the questionnaire survey of villagers' self-governance in twelve villages in five counties of Anhui Province." Fujian Forum (Humanities and Social Sciences Edition) .09(2004):107-110. DOI:CNKI:SUN:FJLW.0.2004-09-023.

[4] Xie Qiushan. "Local government function degradation distance and social public sphere governance dilemma-an analysis based on the case of square dance conflict." Journal of Public Administration 12.03 (2015): 23-32+155-156. doi:10.16149/j.cnki.23-1523.2015.03.003.

[5] Chen J. "The problem of abortion distance of government public cultural services in the perspective of cultural precision poverty alleviation.” Library Forum 37.07 (2017): 74-80.

[6] Chen J. "The problem of abortion distance of government public cultural services in the perspective of cultural precision poverty alleviation.” Library Forum 37.07 (2017): 74-80.

[7] Chen, Qing-Yun. Public policy analysis (2nd edition) [M]. Beijing:Peking University Press, 2011:153.

[8] Xu, Wang. "Public cultural service supply of cultural abortion distance contradiction resolution path." New Century Library .05 (2019):5-9+68. DOI:10.16810/j.cnki.1672-514X.2019.05.001.

[9] Su, Kang. "Research on the evolution and implementation path of China's youth health policy--a perspective based on Edward's policy implementation model. "China Youth Studies .10 (2018):26-32+65.

DOI:10.19633/j.cnki.112579/d.2018.0156.

[10] Chen J. "The problem of abortion distance of government public cultural services in the perspective of cultural precision poverty alleviation"Library Forum 37.07 (2017): 74-80.

[11]Ning, Guoliang. "On public policy implementation bias and its correction." Journal of Hunan University (Social Science Edition) .03(2000):9598.DOI:CNKI:SUN:HDXB.0.2000-03-019.

[12]Qian, Zaijian. "An Inquiry into the Deep Mechanisms Affecting the Subjects of Public Policy Implementation." Theory and Reform .05 (2001):15-17. DOI:CNKI:SUN:LLGG.0.2001-05002. 